\title{
Underestimation of Risk of Carotid Subclinical Atherosclerosis by Cardiovascular Risk Scores in Patients with Psoriatic Arthritis
}

\author{
Jiayun Shen, Steven H. Lam, Qing Shang, Chun-Kwok Wong, Edmund K. Li, Priscilla Wong, \\ Emily W. Kun, Isaac T. Cheng, Martin Li, Tena K. Li, Tracy Y. Zhu, Jack Jock-Wai Lee, \\ Mimi Chang, Alex Pui-Wai Lee, and Lai-Shan Tam
}

ABSTRACT. Objective. To test the performances of established cardiovascular (CV) risk scores in discriminating
subclinical atherosclerosis (SCA) in patients with psoriatic arthritis.
Methods. These scores were calculated: Framingham risk score (FRS), QRISK2, Systematic
COronary Risk Evaluation (SCORE), 10-year atherosclerotic cardiovascular disease risk algorithm
(ASCVD) from the American College of Cardiology and the American Heart Association, and the
European League Against Rheumatism (EULAR)-recommended modified versions (by 1.5 multipli-
cation factor, m-). Carotid intima-media thickness $>0.9 \mathrm{~mm}$ and/or the presence of plaque determined
by ultrasound were classified as SCA+.

Results. We recruited 146 patients [49.4 10.2 yrs, male: 90 (61.6\%)], of whom 142/137/128/118 patients were eligible to calculate FRS/QRISK2/SCORE/ASCVD. Further, 62 (42.5\%) patients were $\mathrm{SCA}+$ and were significantly older, with higher systolic blood pressure and higher low-density lipoprotein cholesterol (all $\mathrm{p}<0.05$ ). All CV risk scores were significantly higher in patients with SCA+ [FRS: 7.8 (3.9-16.5) vs 2.7 (1.1-7.8), p < 0.001; QRISK2: 5.5 (3.1-10.2) vs 2.9 (1.2-6.3), p <0.001; SCORE: 1 (0-2) vs 0 (0-1), p <0.001; ASCVD: 5.6 (2.6-12.4) vs 3.4 (1.4-6.1), p = 0.001]. The Hosmer-Lemeshow test revealed moderate goodness of fit for the $4 \mathrm{CV}$ scores ( $\mathrm{p}$ ranged from 0.087 to 0.686 ). However, of the patients with SCA+, those identified as high risk were only $44.1 \%$ (by FRS > 10\%), 1.8\% (QRISK2 > 20\%), 10.9\% (SCORE > 5\%), and 43.6\% (ASCVD > 7.5\%). By applying the EULAR multiplication factor, 50.8\%/14.3\%/14.5\%/54.5\% of the patients with SCA+ were identified as high risk by m-FRS/m-QRISK2/m-SCORE/m-ASCVD, respectively. EULAR modification increased the sensitivity of FRS and ASCVD in discriminating SCA+ from $44 \%$ to $51 \%$, and $44 \%$ to $55 \%$, respectively.

Conclusion. All CV risk scores underestimated the SCA+ risk. EULAR-recommended modification improved the sensitivity of FRS and ASCVD only to a moderate level. (First Release November 15 2017; J Rheumatol 2018;45:218-26; doi:10.3899/jrheum.170025)

Key Indexing Terms:

PSORIATIC ARTHRITIS

CARDIOVASCULAR DISEASES

ATHEROSCLEROSIS

RISK SCORES

From the Department of Medicine and Therapeutics, The Prince of Wales Hospital, The Chinese University of Hong Kong, Hong Kong, China. Supported by the Health and Medical Research Fund (No. 01120496). $J$. Shen, PhD, Department of Medicine and Therapeutics, The Prince of Wales Hospital, The Chinese University of Hong Kong; S.H. Lam, MSc, Department of Medicine and Therapeutics, The Prince of Wales Hospital, The Chinese University of Hong Kong; Q. Shang, PhD, Department of Medicine and Therapeutics, The Prince of Wales Hospital, The Chinese University of Hong Kong; C.K. Wong, PhD, Department of Chemical Pathology, The Prince of Wales Hospital, The Chinese University of Hong Kong; E.K. Li, MD, Department of Medicine and Therapeutics, The Prince of Wales Hospital, The Chinese University of Hong Kong; P. Wong, MRCP, Department of Medicine and Therapeutics, The Prince of Wales Hospital, The Chinese University of Hong Kong; E.W. Kun, FRCP, Department of Medicine and Geriatrics, Taipo Hospital; I.T. Cheng, BSc, Department of Medicine and Therapeutics, The Prince of Wales Hospital, The Chinese University of Hong Kong; M. Li, PhD, Department of Medicine and

\begin{abstract}
Therapeutics, The Prince of Wales Hospital, The Chinese University of Hong Kong; T.K. Li, BN, Department of Medicine and Therapeutics, The Prince of Wales Hospital, The Chinese University of Hong Kong; T.Y. Zhu, PhD, Bone Quality and Health Center of the Department of Orthopedics and Traumatology, The Prince of Wales Hospital, The Chinese University of Hong Kong; J.J. Lee, PhD, Division of Biostatistics, The Jockey Club School of Public Health and Primary Care, The Chinese University of Hong Kong; M. Chang, MRCP, Department of Medicine and Therapeutics, The Prince of Wales Hospital, The Chinese University of Hong Kong; A.P. Lee, MD, Department of Medicine and Therapeutics, The Prince of Wales Hospital, The Chinese University of Hong Kong; L.S. Tam, MD, Department of Medicine and Therapeutics, The Prince of Wales Hospital, The Chinese University of Hong Kong.

Address correspondence to Dr. L.S. Tam, Department of Medicine and Therapeutics, The Prince of Wales Hospital, The Chinese University of Hong Kong, Shatin, Hong Kong.E-mail: Istam@cuhk.edu.hk Accepted for publication August 30, 2017.
\end{abstract}


Psoriatic arthritis (PsA) is a chronic inflammatory arthritis associated with increased prevalence of cardiovascular (CV) diseases and related mortality ${ }^{1,2,3}$. The increased risk was estimated to be similar to rheumatoid arthritis (RA) ${ }^{4}$. A recent metaanalysis revealed that the $\mathrm{CV}$ morbidity was increased by $43 \%$ in patients with PsA, compared with the general population ${ }^{5}$. Subclinical atherosclerosis (SCA), which is a good surrogate endpoint for $\mathrm{CV}$ disease in the general population $^{6,7}$ and in patients with $\mathrm{RA}^{8,9,10}$, is also more common in patients with PsA ${ }^{11,12}$. The higher prevalence of traditional CV risk factors in patients with PsA probably contributed to the increased risk ${ }^{3,13}$. Nevertheless, abnormally increased carotid intima-media thickness (IMT) was observed in PsA patients without CV events or classic CV disease risk factors when compared with matched controls ${ }^{14}$. Moreover, uncontrolled low-grade inflammation also accounts for the $24 \%$ increased risk of major adverse CV events in patients with PsA who were not prescribed a disease-modifying antirheumatic drug (DMARD) compared with control subjects in a population-based cohort study ${ }^{1}$. Nonsteroidal antiinflammatory drugs and glucocorticoid are commonly prescribed drugs in the treatment of PsA; both are associated with increased $\mathrm{CV}$ risks ${ }^{15}$. Thus, stratification of $\mathrm{CV}$ risks is more complicated in patients with PsA.

In the general population, certain predictive scores were developed to estimate CV risks. The Framingham risk score (FRS) ${ }^{16}$, QRISK2 ${ }^{17}$, Systematic COronary Risk Evaluation $(\mathrm{SCORE})^{18}$, and the 10 -year atherosclerotic $\mathrm{CV}$ disease risk algorithm (ASCVD) ${ }^{19}$ from the American College of Cardiology and the American Heart Association (ACC/AHA) are among the most widely used $\mathrm{CV}$ risk scores. In RA, these risk scores generally underestimated the risk of $\mathrm{CV}$ events ${ }^{20}$ or $\mathrm{SCA}^{21,22,23,24}$. A 1.5 multiplication factor for the risk scores is recommended by the European League Against Rheumatism (EULAR) in patients with RA who fulfill 2 out of 3 of the following criteria: disease duration $>10$ years; rheumatoid factor and/or anticyclic citrullinated peptide positivity; and presence of extraarticular manifestations ${ }^{25}$. However, the performance was still unsatisfactory with this modification $21,22,23,26$.

In PsA, similar data are scarce. Ernste, et $a^{27}$ and Eder, et $a l^{28}$ suggested that FRS underestimated the risk of $\mathrm{CV}$ events and SCA in PsA, respectively. Gulati and colleagues found that SCORE could not explain the increased prevalence of established CV diseases in patients with PsA compared with the general population ${ }^{29}$. Comprehensive evaluation and comparison of the risk scores in patients with PsA is lacking. Moreover, because the increased $\mathrm{CV}$ risk in PsA was estimated to be similar to $\mathrm{RA}^{4}$, it would be important to ascertain whether the multiplication factor of 1.5 should be introduced to patients with PsA to refine CV risk assessment.

In our study, we aimed to evaluate the performance of FRS, QRISK2, SCORE, and ASCVD in discriminating carotid SCA in patients with PsA. We also tested whether introducing the multiplication factor of 1.5 could improve their performance.

\section{MATERIALS AND METHODS}

Patients. Data from 162 patients with PsA who fulfilled the Classification for Psoriatic Arthritis criteria ${ }^{30}$ and who underwent carotid ultrasound were retrieved in our study. There were 93 patients who participated in a previous cohort study ${ }^{11,31}$, and 69 patients were enrolled in an ongoing prospective study aiming to assess the effect of treat-to-target in the prevention of atherosclerosis progression (trial registration no.: NCT02232321). In these 2 studies, patients with established CV diseases (e.g., myocardial infarction, angina, stroke, transient ischemic attack, etc.) or clinically significant renal disease (serum creatinine level $>270 \mathrm{~mol} / \mathrm{l}$ ) were excluded. There were 16 patients excluded because of a lack of information for calculating at least 1 $\mathrm{CV}$ risk score. There were 146 patients ( 79 and 67 from the 2 studies, respectively) who were finally included in the analysis. Ethics approval was obtained from the Ethics Committee of The Chinese University of Hong Kong-New Territories East Cluster Hospitals (reference number: CRE-2012.478), and written informed consent was obtained from all participants according to the Declaration of Helsinki.

Clinical interview. Pain, as well as physicians' and patients' global assessments, were evaluated using a 100-point visual analog scale, where 0 indicated excellent well-being and 100 indicated feeling extremely unwell. Physical examination included the number of tender and swollen joints using the 68 tender/66 swollen joint count, the presence of dactylitis, and the number of permanently deformed joints. The Health Assessment Questionnaire was used to evaluate physical function, and the psoriasis area and severity index was used to assess the extent of skin involvement ${ }^{32}$. Overall disease activity was assessed using disease activity in psoriatic arthritis (DAPSA) ${ }^{33}$, and patients were considered as being in remission (REM, DAPSA $\leq 4)$, or having low (LDA, $>4$ DAPSA $\leq 4)$, moderate $($ MDA,$>14$ DAPSA $\leq 28)$, or high (HAD, DAPSA > 28) disease activity ${ }^{34}$. Anthropometric measurements including height, weight, and 2 consecutive blood pressure (BP) readings in sitting position were also recorded. Body mass index (BMI) was calculated. Other data obtained from patients with PsA through the interview and chart review included smoking habits, history of diabetes, hypertension, and hypercholesterolemia. Drug history was retrieved from case notes or obtained during the clinical assessment. All patients were interviewed and examined using standardized data collection instruments. Checks were done for complete blood count, liver and renal function, erythrocyte sedimentation rate, C-reactive protein (CRP), fasting blood glucose, and lipid profile [total cholesterol (TC), high density lipoprotein-cholesterol, low density lipoprotein-cholesterol (LDL), and triglycerides (TGC)].

Carotid atherosclerosis. Carotid IMT was measured at 6 carotid arterial segments using a high-resolution B-mode ultrasound machine (iE33, Philips) by an experienced cardiologist (QS) as previously described ${ }^{35,36}$. Briefly, duplex carotid ultrasound was performed using an 11-MHz linear vascular probe. The IMT was measured offline in the distal common carotid artery (the arterial segment $1 \mathrm{~cm}$ proximal to the carotid bulb), bulb, and proximal internal carotid artery (the arterial segment $1 \mathrm{~cm}$ distal to the carotid bifurcation) using dedicated software (QLab 6.0, Philips), and was analyzed by the same investigator, who was blinded to all clinical information. The IMT values of 6 arterial segments were measured, the maximum of which were calculated for further analysis. Plaque was defined as a localized thickening $>1.2 \mathrm{~mm}^{11,36,37}$. Our study involved a single ultrasonographer and a single reader. The intraclass correlation coefficient for the mean of the site-specific IMT values was $0.97^{11,35}$. Patients with maximum IMT $>0.9 \mathrm{~mm}$ and/or the presence of plaque were classified as $\mathrm{SCA}+{ }^{21,22}$.

Risk score calculation. FRS was calculated for Framingham 10-year risk of general CV disease ${ }^{16}$. QRISK2 was calculated by the QRISK2-2016 risk calculator ${ }^{17}$. SCORE was calculated by the interactive software HeartScore Risk Calculator $1.0^{18}$, developed by the European Society of Cardiology.

Personal non-commercial use only. The Journal of Rheumatology Copyright @ 2018 . All rights reserved. 
There are no charts designed for Asians; however, because the Chinese population in general has a lower $\mathrm{CV}$ risk ${ }^{38,39}$, the European low-risk chart was adopted. ASCVD risk estimator was used to calculate atherosclerotic CV disease risk ${ }^{19}$. EULAR modified scores (multiplied by 1.5 ) were calculated for all risk scores and labeled with the prefix "m-". Patients with FRS $>10 \%{ }^{24}$, QRISK2 $>20 \%{ }^{22}$, SCORE $>5 \%^{22}$, and ASCVD $>7.5 \% 22$ were considered as having high $\mathrm{CV}$ risks according to the $\mathrm{CV}$ risk scores.

Statistical analysis. Results are expressed as mean \pm SD or median (interquartile range) as appropriate. Comparisons between 2 groups were assessed using the Student $t$ test or Mann-Whitney U test for continuous variables, and chi-square test for categorical variables. The receiver-operating characteristic (ROC) curve was used to evaluate the performance of $\mathrm{CV}$ risk scores in discriminating carotid atherosclerosis. Cutoff values of the $\mathrm{CV}$ risk scores with best combined sensitivity and specificity were determined according to the Youden index. The Hosmer-Lemeshow test was applied to assess the goodness of fit for the observed and expected SCA case numbers estimated by the CV risk scores. Multivariate logistic regression was used to identify the independent risk factor for carotid atherosclerosis in patients with PsA. All traditional CV risk factors with $p<0.1$ in the univariate analysis were included in the multivariate analysis as potential confounding factors. All statistical analyses were conducted using IBM SPSS Statistics Version 22. A minimal level of significance of $\mathrm{p}<0.05$ was used.

\section{RESULTS}

Patients' characteristics and CV risk scores. There were 146 patients with PsA [90 male (61.6\%), mean \pm SD age: $49.4 \pm$ $10.2 \mathrm{yrs}$ ] included. Median disease duration was 7 years and ranged from 1 month to 40 years. There were 7 (4.8\%) patients in REM, 64 (43.8\%) with LDA, 52 (35.6\%) with MDA, and 23 (15.8\%) with HDA. Traditional CV risk factors were common: $54(37.0 \%)$ patients were overweight (BMI $\geq 25 \mathrm{~kg} / \mathrm{m}^{2}$ ) and $25(17.1 \%)$ patients were obese (BMI $\geq 30$ $\left.\mathrm{kg} / \mathrm{m}^{2}\right) ; 42$ patients $(28.8 \%)$ were current or ever smokers; and $64(43.8 \%), 19(13.0 \%)$, and 17 (11.6\%) had hypertension, diabetes, and dyslipidemia, respectively. Overall, 116 patients (79.5\%) had at least 1 of these traditional risk factors. Data for $142,137,128$, and 118 patients were available to calculate FRS, QRISK2, SCORE, and ASCVD, respectively. The following patients were classified as having high CV risk: $42(29.6 \%)$ by FRS, 2 (1.5\%) by QRISK2, $6(4.7 \%)$ by SCORE, and 35 (29.7\%) by ASCVD. More details of the patients' characteristics were shown in Table 1.

$S C A$ and $C V$ risk scores. The mean IMT of the 6 sites was $0.70 \pm 0.12 \mathrm{~mm}$, and maximum IMT was $0.85 \pm 0.18 \mathrm{~mm}$. Respectively, 8 patients $(5.5 \%)$ and $42(28.8 \%)$ had mean and maximum IMT $>0.9 \mathrm{~mm}$. Thirty-three $(22.6 \%)$ patients had at least 1 carotid plaque. There were $62(42.5 \%)$ patients defined as SCA+ with maximum IMT $>0.9 \mathrm{~mm}$ and/or the presence of plaque. The patients with SCA+ were significantly older $(53.3 \pm 9.0$ vs $47.0 \pm 10.2$ years, $\mathrm{p}<0.001)$, had higher systolic BP $(137 \pm 25$ vs $129 \pm 17$ $\mathrm{mmHg}, \mathrm{p}=0.018)$, TC $(5.3 \pm 0.9$ vs $4.9 \pm 0.9 \mathrm{mmol} / \mathrm{l}$, $\mathrm{p}=0.007)$, and LDL levels ( $3.2 \pm 0.8$ vs $2.9 \pm 0.8 \mathrm{mmol} / \mathrm{l}$, $\mathrm{p}=0.015)$. There was a trend suggesting increased TGC and fasting glucose levels in patients with SCA+ (Table 2). All CV risk scores were significantly higher in patients with SCA $+(p \leq 0.001$, Table 2$)$. The cases of diabetes and
Table 1. Clinical characteristics of all patients. Values are median (interquartile range) or mean \pm SD unless otherwise specified.

\begin{tabular}{lc}
\hline Variables & All Patients, $\mathrm{n}=146$ \\
\hline Male, $\mathrm{n}(\%)$ & $90(61.6)$ \\
Age, yrs & $49.4 \pm 10.2$ \\
PsA characteristics & \\
PsA disease duration, yrs & $7.0(3.2-13.0)$ \\
Tender joint count, 0-68 & $3(0-7)$ \\
Swollen joint count, 0-66 & $1(0-2)$ \\
Damaged joint count, $0-68$ & $2(0-5)$ \\
VAS pain, 0-100 & $40(20-60)$ \\
PtGA, 0-100 & $50(30-60)$ \\
PGA, 0-100 & $20(10-40)$ \\
PASI, 0-72 & $3.5(1.0-8.6)$ \\
HAQ, 0-3 & $0.38(0-0.88)$ \\
ESR, mm/h & $22(11-37)$ \\
CRP, mg/dl & $0.4(0.2-1.2)$ \\
DAPSA, 0-164 & $14.9(9.0-21.2)$ \\
PsA medication, $\mathrm{n}(\%)$ & \\
NSAID & $71(49.0)$ \\
Steroid & $10(6.9)$ \\
Synthetic DMARD & $68(46.9)$ \\
Biologic DMARD & $10(6.9)$ \\
CV risk factors & \\
BMI, kg/m ${ }^{2}$ & $26.1 \pm 4.9$ \\
Ever smoker, $\mathrm{n}(\%)$ & $42(28.8)$ \\
Systolic BP, mmHg & $132 \pm 21$ \\
Diastolic BP, mmHg & $82 \pm 12$ \\
Total cholesterol, mmol/1 & $5.1 \pm 0.9$ \\
HDL cholesterol, mmol/1 & $1.5 \pm 0.5$ \\
LDL cholesterol, mmol/1 & $3.0 \pm 0.8$ \\
Triglycerides, mmol/1 & $1.5 \pm 0.9$ \\
Fasting glucose, mmol/1 & $5.4 \pm 1.2$ \\
Hypertension, $\mathrm{n}(\%)$ & $64(43.8)$ \\
Diabetes, $\mathrm{n}(\%)$ & $19(13.0)$ \\
Dyslipidemia, $\mathrm{n}(\%)$ & $17(11.6)$ \\
CV risk scores*, $\mathrm{n}(\%)$ & \\
FRS & \\
QRISK2 & \\
SCORE & \\
ASCVD & \\
\hline
\end{tabular}

*Available in 142, 137, 128, and 118 patients for FRS, QRISK2, SCORE, and ASCVD, respectively. PsA: psoriatic arthritis; VAS: visual analog scale; PtGA: patient's global assessment; PGA: physician's global assessment; PASI: Psoriasis Area and Severity Index; HAQ: Health Assessment Questionnaire; ESR: erythrocyte sedimentation rate; CRP: C-reactive protein; DAPSA: Disease Activity Index for Psoriatic Arthritis; NSAID: nonsteroidal antiinflammatory drugs; DMARD: disease-modifying antirheumatic drugs; CV: cardiovascular; BMI: body mass index; BP: blood pressure; HDL: high-density lipoprotein; LDL: low-density lipoprotein; FRS: Framingham risk score; SCORE: Systematic COronary Risk Evaluation; ASCVD: atherosclerotic cardiovascular disease risk algorithm.

dyslipidemia were higher in patients with SCA- but the difference between SCA+ and SCA- was not significant (Table 2). The areas under the ROC curve (AUROC) for discriminating SCA+ were $0.72(95 \%$ CI $0.63-0.80$, $\mathrm{p}<0.001)$ for FRS, $0.69(0.60-0.77, \mathrm{p}<0.001)$ for QRISK2, $0.67(0.58-0.77 ; \mathrm{p}=0.001)$ for SCORE, and $0.67(0.58-0.77 ; \mathrm{p}=0.001)$ for ASCVD (Figure 1). The

Personal non-commercial use only. The Journal of Rheumatology Copyright @ 2018. All rights reserved 
Table 2. Clinical characteristics of patients with and without subclinical atherosclerosis. Values are median (interquartile range) or mean \pm SD unless otherwise specified.

\begin{tabular}{|c|c|c|c|}
\hline & $\mathrm{SCA}_{-}, \mathrm{n}=84$ & $\mathrm{SCA}+, \mathrm{n}=62$ & $\mathrm{p}$ \\
\hline Male, n (\%) & $49(58.3)$ & $41(66.1)$ & 0.338 \\
\hline Age, yrs & $47.0 \pm 10.2$ & $53.3 \pm 9.0$ & $<0.001$ \\
\hline \multicolumn{4}{|l|}{ PsA characteristics } \\
\hline PsA disease duration, yrs & $8.2(3.0-13.3)$ & $5.9(3.4-11.3)$ & 0.606 \\
\hline Tender joint count, $0-68$ & $2(0-6)$ & $5(1-8)$ & 0.148 \\
\hline Swollen joint count, $0-66$ & $1(0-2)$ & $1(0-2)$ & 0.687 \\
\hline Damaged joint count, $0-68$ & $1(0-5)$ & $2(0-6)$ & 0.624 \\
\hline VAS pain, $0-100$ & $40(20-60)$ & $50(30-70)$ & 0.388 \\
\hline PtGA, 0-100 & $50(30-60)$ & $50(40-70)$ & 0.113 \\
\hline PGA, 0-100 & $20(10-35)$ & $30(10-40)$ & 0.112 \\
\hline PASI, 0-72 & $3.5(1.4-8.4)$ & $3.4(1.0-8.7)$ & 0.768 \\
\hline HAQ, 0-3 & $0.38(0-0.88)$ & $0.31(0-0.88)$ & 0.874 \\
\hline $\mathrm{ESR}, \mathrm{mm} / \mathrm{h}$ & $20(10-37)$ & $25(13-37)$ & 0.327 \\
\hline $\mathrm{CRP}, \mathrm{mg} / \mathrm{dl}$ & $0.4(0.2-1.2)$ & $0.4(0.1-1.3)$ & 0.946 \\
\hline DAPSA, $0-164$ & $14.2(8.2-20.2)$ & $16.4(9.4-21.5)$ & 0.182 \\
\hline \multicolumn{4}{|l|}{ PsA medication, $\mathrm{n}(\%)$} \\
\hline NSAID & $44(53.0)$ & $27(43.5)$ & 0.259 \\
\hline Steroid & $6(7.1)$ & $4(6.5)$ & 0.870 \\
\hline Synthetic DMARD & $41(49.4)$ & $27(43.5)$ & 0.485 \\
\hline Biologic DMARD & $5(6.0)$ & $5(8.1)$ & 0.631 \\
\hline \multicolumn{4}{|l|}{$\mathrm{CV}$ risk factors } \\
\hline BMI, $\mathrm{kg} / \mathrm{m}^{2}$ & $26.3 \pm 5.2$ & $25.8 \pm 4.5$ & 0.496 \\
\hline Ever smoker, n (\%) & $21(25.0)$ & $21(33.9)$ & 0.242 \\
\hline Systolic BP, mmHg & $129 \pm 17$ & $137 \pm 25$ & 0.018 \\
\hline Diastolic BP, mmHg & $81 \pm 12$ & $83 \pm 11$ & 0.372 \\
\hline Total cholesterol, mmol/1 & $4.9 \pm 0.9$ & $5.3 \pm 0.9$ & 0.007 \\
\hline HDL cholesterol, mmol/l & $1.5 \pm 0.5$ & $1.5 \pm 0.4$ & 0.939 \\
\hline LDL cholesterol, mmol/1 & $2.9 \pm 0.8$ & $3.2 \pm 0.8$ & 0.015 \\
\hline Triglycerides, mmol/1 & $1.4 \pm 0.9$ & $1.6 \pm 1.0$ & 0.084 \\
\hline Fasting glucose, $\mathrm{mmol} / \mathrm{l}$ & $5.2 \pm 0.9$ & $5.6 \pm 1.5$ & 0.068 \\
\hline Hypertension, $\mathrm{n}(\%)$ & $36(42.9)$ & $28(45.2)$ & 0.782 \\
\hline Diabetes, $\mathrm{n}(\%)$ & $11(13.1)$ & $8(12.9)$ & 0.973 \\
\hline Dyslipidemia, n (\%) & 10 (11.9) & $7(11.3)$ & 0.909 \\
\hline \multicolumn{4}{|l|}{$\mathrm{CV}$ risk scores", $\mathrm{n}(\%)$} \\
\hline FRS & $2.7(1.1-7.8)$ & $7.8(3.9-16.5)$ & $<0.001$ \\
\hline QRISK2 & $2.9(1.2-6.3)$ & $5.5(3.1-10.2)$ & $<0.001$ \\
\hline SCORE & $0(0-1)$ & $1(0-2)$ & $<0.001$ \\
\hline ASCVD & $3.4(1.4-6.1)$ & $5.6(2.6-12.4)$ & 0.001 \\
\hline
\end{tabular}

*Total number of SCA \pm patients with available scores: 83/59, 81/56, 73/55, and 63/55, for FRS, QRISK2, SCORE, and ASCVD, respectively. PsA: psoriatic arthritis; SCA: subclinical atherosclerosis; VAS: visual analog scale; PtGA: patient's global assessment; PGA: physician's global assessment; PASI: Psoriasis Area and Severity Index; HAQ: Health Assessment Questionnaire; ESR: erythrocyte sedimentation rate; CRP: C-reactive protein; DAPSA: Disease Activity Index for Psoriatic Arthritis; NSAID: nonsteroidal antiinflammatory drugs; DMARD: disease-modifying antirheumatic drugs; CV: cardiovascular; BMI: body mass index; BP: blood pressure; HDL: high-density lipoprotein; LDL: low-density lipoprotein; FRS: Framingham risk score; SCORE: Systematic COronary Risk Evaluation; ASCVD: atherosclerotic cardiovascular disease risk algorithm.

Hosmer-Lemeshow test revealed moderate goodness of fit for the $4 \mathrm{CV}$ scores (p ranging from 0.087 to 0.686 , Figure 2). Sensitivity and specificity of the cutoff values with the highest Youden index was shown in Table 3.

By applying the preset cutoff values, $44.1 \%, 1.8 \%, 10.9 \%$, and $43.6 \%$ of the patients with SCA+ were identified as high risk by FRS, QRISK2, SCORE, and ASCVD, respectively (Figure 3). There were $19.3 \%$ and $17.5 \%$ of patients with SCA- also classified as high risk by FRS and ASCVD.
EULAR modification of the CV risk scores. While the 1.5 multiplication factor was introduced to the risk scores, $50.8 \%, 14.3 \%, 14.5 \%$, and $54.5 \%$ of the patients with SCA+ were identified as high risk by m-FRS, m-QRISK2, $\mathrm{m}$-SCORE, and m-ASCVD, respectively (Figure 3). The patients with SCA- who were classified as high risk also increased to $30.1 \%$ (m-FRS), $7.4 \%$ (m-QRISK2), $4.1 \%$ (m-SCORE), and 36.5\% (m-ASCVD). Sensitivity and specificity of the modified cutoff values were shown in Table 3 .

Personal non-commercial use only. The Journal of Rheumatology Copyright $\odot$ 2018. All rights reserved. 

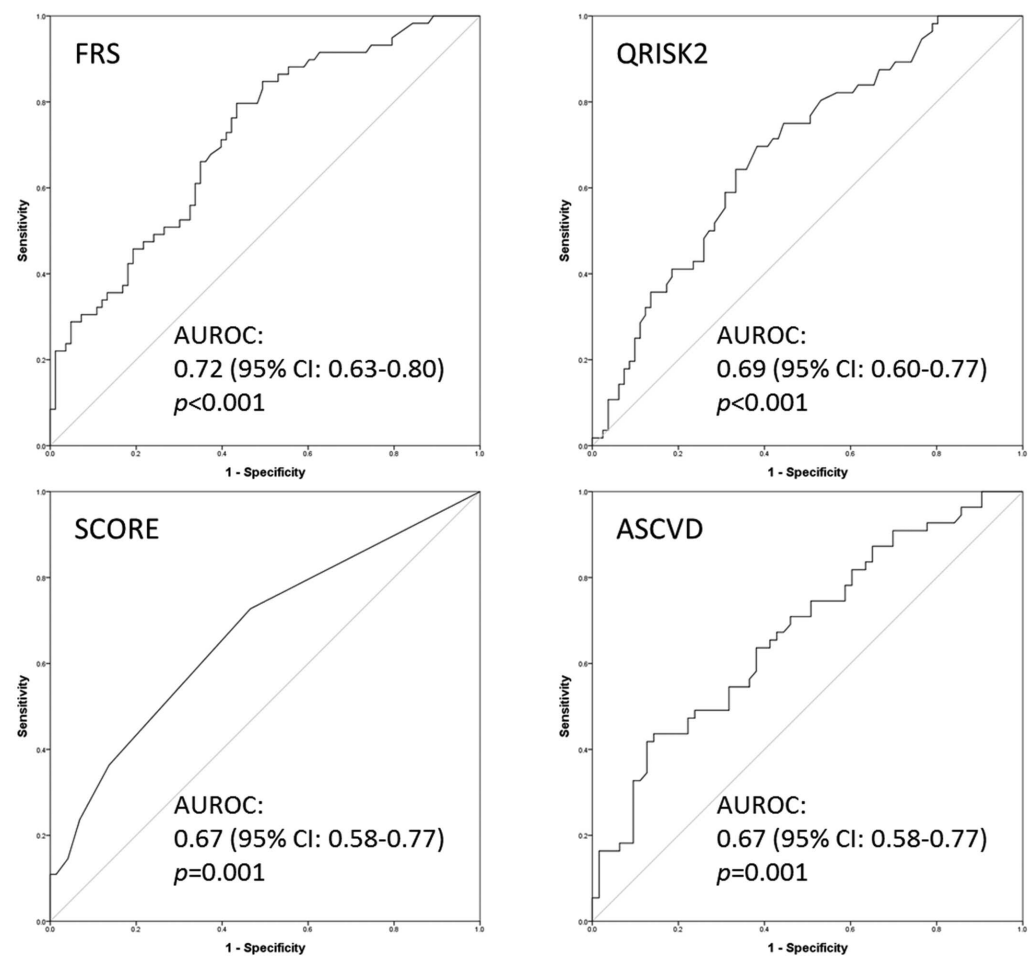

Figure 1. Receiver-operating characteristic curve of the risk scores in discriminating subclinical atherosclerosis. AUROC: area under receiver-operating characteristic curve; FRS: Framingham risk score; SCORE: Systematic COronary Risk Evaluation; ASCVD: atherosclerotic cardiovascular disease risk algorithm.
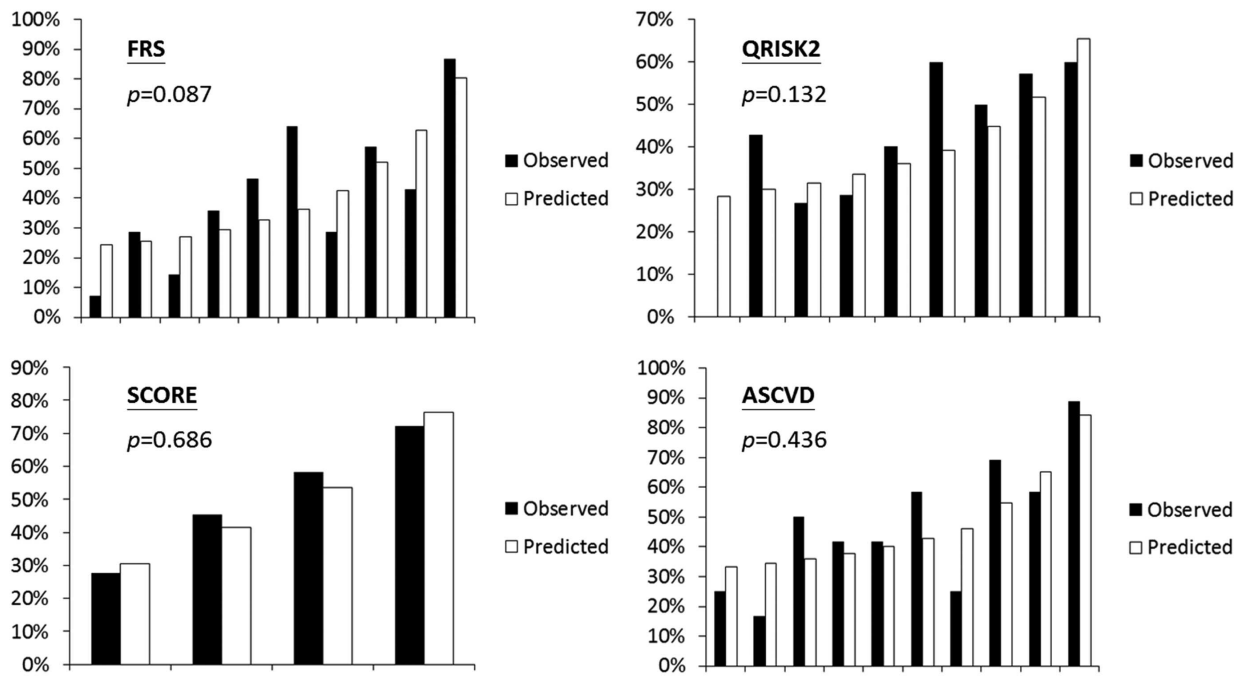

Figure 2. Hosmer-Lemeshow test for the goodness of fit for the observed and expected risk of subclinical atherosclerosis by the risk scores. FRS: Framingham risk score; SCORE: Systematic COronary Risk Evaluation; ASCVD: atherosclerotic cardiovascular disease risk algorithm.

Independent predictors for SCA among traditional CV risk factors. Age, systolic BP, LDL, TGC, and fasting glucose were included in a multivariate logistic regression model to identify the independent predictors for SCA+. Older age (OR 1.090, 95\% CI 1.044-1.138; $p<0.001$ ) and higher
LDL level (OR 1.899, 95\% CI 1.160-3.108; p = 0.011) were found to be independently associated with SCA+. None of the $4 \mathrm{CV}$ risk scores were independently associated with $\mathrm{SCA}+$ if they were also included in the regression model. 
Table 3. Sensitivity and specificity of preset and modified cutoffs for risk scores, and cutoffs with highest overall accuracy. Values are $\%$.

\begin{tabular}{lccc}
\hline Risk Scores & Cutoff & Sensitivity & Specificity \\
\hline Highest Youden index & & & \\
FRS & 3.7 & 79.7 & 56.6 \\
QRISK2 & 3.8 & 69.6 & 61.7 \\
SCORE & 0.5 & 72.7 & 53.4 \\
ASCVD & 8.3 & 43.6 & 85.7 \\
Preset & & & \\
FRS & 10 & 44.1 & 80.7 \\
QRISK2 & 20 & 1.8 & 98.8 \\
SCORE & 5 & 10.9 & 98.6 \\
ASCVD & 7.5 & 43.6 & 82.5 \\
Modified & & & \\
m-FRS & 10 & 50.8 & 69.9 \\
m-QRISK2 & 20 & 14.3 & 93.8 \\
m-SCORE & 5 & 14.5 & 95.9 \\
m-ASCVD & 7.5 & 54.5 & 65.1 \\
\hline
\end{tabular}

FRS: Framingham risk score; SCORE: Systematic COronary Risk Evaluation; ASCVD: atherosclerotic cardiovascular disease risk algorithm; m-: modified.

\section{DISCUSSION}

This is the first study, to our knowledge, that comprehensively evaluated the performance of established CV risk assessment tools in patients with PsA. Our results suggested that all $4 \mathrm{CV}$ risk assessment tools, including FRS, QRISK2, SCORE, and ASCVD, exhibited moderate discrimination between PsA patients with or without carotid SCA. However, the preset cutoff values of all scores underestimated the risk of SCA. The 1.5 multiplication factor only led to a modest improvement in their performance.

All the CV risk scores were calculated mainly based on traditional CV risk factors, and were developed for the CV risk stratification in the general population. Patients with PsA had higher prevalence of SCA compared with controls even after adjustment of the traditional $\mathrm{CV}$ risk factors ${ }^{11}$. It is not unexpected that the risk scores would underestimate the $\mathrm{CV}$ risk in patients with PsA. In RA, although 1 study suggested that QRISK2 may have overestimated the CV risk ${ }^{20}$, most studies found CV risk was underestimated by $\mathrm{FRS}^{20,22,24}$, SCORE $^{20,21,22,23,26}$, or ASCVD ${ }^{22,24}$. In PsA, FRS ${ }^{27,28}$ and $\mathrm{SCORE}^{29}$ had also been reported to underestimate CV risk. In our study, PsA patients with SCA had significantly higher $\mathrm{CV}$ risks evaluated by all $4 \mathrm{CV}$ risk assessment tools ( $\mathrm{p} \leq$ $0.001)$. All scores had moderate discriminating abilities in SCA (AUROC ranged from 0.67 to 0.72 ), and moderate goodness of fit by the Hosmer-Lemeshow test ( $p>0.05)$. However, by applying the preset cutoff values (FRS $>10 \%$, QRISK2 > 20\%, SCORE > 5\%, ASCVD > 7.5\%), 55.9\%, $98.2 \%, 89.1$, and $56.4 \%$ of the patients with SCA+ were classified as "low risk" according to FRS, QRISK2, SCORE, and ASCVD, respectively. This discrepancy between the moderate discriminating abilities of the 4 scores (as continuous variables) and poor calibrating abilities of the cutoff values could be explained by the threshold selection. As shown in Table 3, the cutoff values with best accuracy (highest Youden index) were much lower in FRS, QRISK2, and SCORE compared with the preset ones. Only the preset cutoff value for ASCVD was close to the cutoff value with best accuracy.

For CV risk management in RA, EULAR recommends applying a multiplication factor of 1.5 to SCORE in selected patients to enhance the risk estimates ${ }^{25}$. However, the improvement of risk assessment by such modification appeared to be suboptimal 21,22,23,26. Based on the knowledge that patients with PsA had similar $\mathrm{CV}$ risk compared to patients with $\mathrm{RA}^{4}$, we also tested whether the multiplication factor would improve the performance of the scores for the first time. By adopting the multiplication factor, although the sensitivity of the various $\mathrm{CV}$ assessment tools increased by $6.7 \%$ to $12.5 \%$, a significant proportion of patients with $\mathrm{SCA}+$ were still misclassified as having "low risk" (over $45 \%$ by $m-F R S$ and $m-A S C V D$, and over $85 \%$ by m-QRISK 2 and $\mathrm{m}$-SCORE, Figure 3). This result is not surprising because adopting the multiplication factor of 1.5 is the same as lowering the cutoff values to $6.7 \%, 13.3 \%, 3.3 \%$, and $5 \%$ for FRS, QRISK2, SCORE, and ASCVD, respectively. Those are still not close to the cutoffs with best accuracy.

A possible explanation for the underestimation of the SCA risk is chronic inflammation in patients with PsA. Low-grade inflammation may account for almost a quarter of the increased $\mathrm{CV}$ risk in patients with PsA who were not prescribed DMARD ${ }^{1}$, and systemic therapy significantly decreased the $\mathrm{CV}$ risk in patients with psoriatic disease ${ }^{15}$. The inflammatory process is believed to enhance the expression of adhesion molecules, and promote endothelial cell dysfunction and smooth muscle cell proliferation, leading to atherosclerotic plaque formation ${ }^{40}$. Proinflammatory cytokines also play a role in plaque remodeling and fibrous cap thinning, increasing plaque vulnerability ${ }^{40}$. Targeted antiinflammatory therapy by blocking tumor necrosis factor halted, or even reversed the progression of IMT in patients with inflammatory arthritis ${ }^{41}$. While atherosclerosis is an inflammatory disease ${ }^{40,42}$, most $\mathrm{CV}$ risk scores do not include inflammatory biomarkers. Among all $4 \mathrm{CV}$ risk assessment tools, only RA is included as one of the CV risk factors in QRISK2. Assuming patients with PsA had similar CV risk compared to patients with RA, we had recalculated the $\mathrm{CV}$ risk by marking this question as "yes" in our PsA cohort, and the performance was similar (AUROC changed from 0.685 to 0.687 , detailed data not shown). The Reynolds Risk Score (RRS) includes high-sensitivity CRP as one of the CV risk factors ${ }^{43,44}$. Because RRS is limited to nondiabetic subjects and only 90 patients with PsA in our cohort provided adequate data to calculate RRS, we did not include RRS in the main analysis. However, the discriminating ability of RRS in SCA was even lower than any other risk scores [AUROC: $0.66(0.54-0.77) ; \mathrm{p}=0.010$ ].

Personal non-commercial use only. The Journal of Rheumatology Copyright $\odot$ 2018. All rights reserved. 
a.

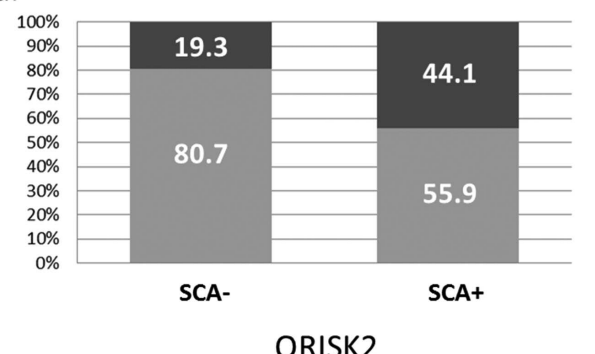

QRISK2
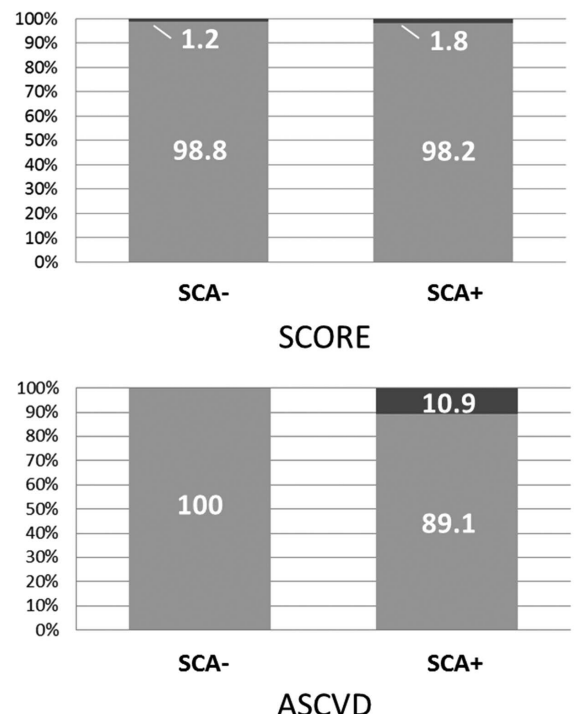

ASCVD

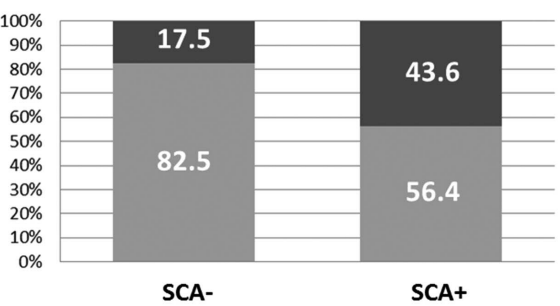

b.
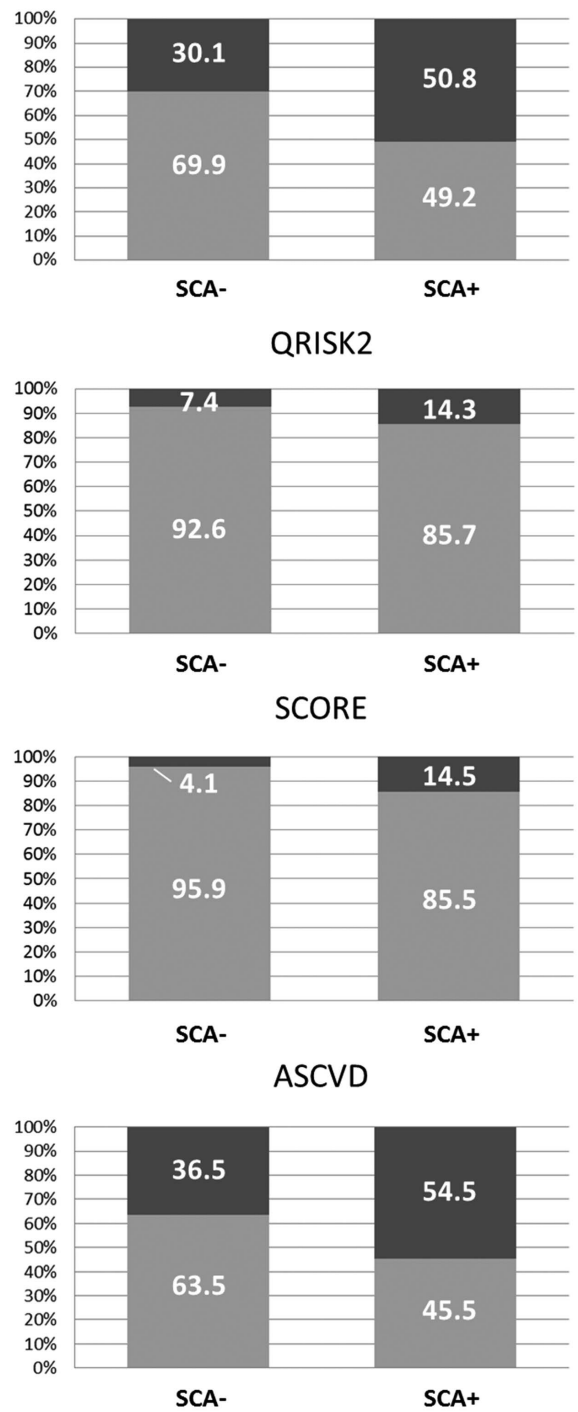

Figure 3. Original risk scores and EULAR modified risk scores in discriminating subclinical atherosclerosis using the preset cutoffs. (A) Original risk scores. (B) EULAR modified risk scores. Dark grey: high risk; Light grey: low risk. High risk: FRS > 10\%, QRISK2 > 20\%, SCORE > 5\%, and ASCVD > 7.5\%. SCA: subclinical atherosclerosis; FRS: Framingham risk score; SCORE: Systematic COronary Risk Evaluation; ASCVD: atherosclerotic cardiovascular disease risk algorithm; EULAR: European League Against Rheumatism.

To overcome this problem, some authors suggested lowering the threshold for high CV risk in patients with $\mathrm{RA}^{22,24}$. Dessein, et al demonstrated that FRS and SCORE effectively estimated the presence of carotid plaques in patients with RA at very low threshold values of $7.3 \%-10.8 \%$, and $0.5 \%-1.5 \%$, respectively ${ }^{45}$. According to our results, it would be more important to improve the AUROC to increase the overall predictive accuracy. Arts, et $a l^{26}$ tried to incorporate RA disease activity (if 28-joint Disease Activity Score > 5.1) into the SCORE; however, the adapted SCORE algorithm did not provide sufficient improvement in $\mathrm{CV}$ risk prediction in patients with RA.
Nevertheless, the usefulness of a similar adaptation of the SCORE algorithm in PsA would need to be addressed in future large cohort studies.

The strength of our study was the comprehensive CV risk assessment and ultrasonographic evaluation. We had thoroughly evaluated 6 sites of carotid arteries by a single investigator, which ensured the identification of SCA. Our study also has a few limitations. First, the risk scores were originally developed in American or European communities; therefore, the CV risk calculated may be overestimated or underestimated because of differences in ethnicity. Second, our results may not be applicable to patients with PsA from

Personal non-commercial use only. The Journal of Rheumatology Copyright $@$ ( 2018. All rights reserved. 
other ethnic backgrounds. Third, the outcome of our study is only a surrogate of clinical CV events. The performance of the risk scores in predicting $\mathrm{CV}$ events should be evaluated in future prospective studies. Fourth, our sample size is small; further validation is necessary to consolidate the conclusion. Nonetheless, the correlation of SCA and high CV risk is well recognized. In studies based on the general population, the 10 -year risk of coronary heart disease ranged from $11 \%$ to $25 \%$ in patients with carotid plaque or increased IMT, while in patients without SCA, it ranged only from $1 \%$ to $8 \% \%^{6,46,47,48}$. Fourth, the addition of carotid IMT measurements to the risk stratification may be modest ${ }^{49}$, and plaque may be more robust in predicting $\mathrm{CV}$ events ${ }^{50}$. Nevertheless, in a subgroup analysis considering only patients with carotid plaque as SCA+, the results were generally similar (AUROC ranged from 0.626 to 0.664 , $\mathrm{p}$ ranged from 0.026 to 0.004 ; over half the patients with SCA+ were missed by FRS $>10 \%$ and ASCVD $>7.5 \%$, and over $80 \%$ were missed by QRISK 2 $>20 \%$ and SCORE $>5 \%$ ). Finally, carotid plaque burden and vulnerability were not assessed in our study.

FRS, QRISK2, SCORE, and ASCVD may underestimate the risk of SCA in patients with PsA. The 1.5 multiplication factor provided limited improvement in the performance of these CV risk assessment tools. Disease-specific CV risk prediction algorithms should be developed.

\section{REFERENCES}

1. Ogdie A, Yu Y, Haynes K, Love TJ, Maliha S, Jiang Y, et al. Risk of major cardiovascular events in patients with psoriatic arthritis, psoriasis and rheumatoid arthritis: a population-based cohort study. Ann Rheum Dis 2015;74:326-32.

2. Gladman DD, Ang M, Su L, Tom BD, Schentag CT, Farewell VT. Cardiovascular morbidity in psoriatic arthritis. Ann Rheum Dis 2009;68:1131-5.

3. Han C, Robinson DW Jr, Hackett MV, Paramore LC, Fraeman KH, Bala MV. Cardiovascular disease and risk factors in patients with rheumatoid arthritis, psoriatic arthritis, and ankylosing spondylitis. J Rheumatol 2006;33:2167-72.

4. Jamnitski A, Symmons D, Peters MJ, Sattar N, McInnes I, Nurmohamed MT. Cardiovascular comorbidities in patients with psoriatic arthritis: a systematic review. Ann Rheum Dis 2013;72:211-6.

5. Polachek A, Touma Z, Anderson M, Eder L. Risk of cardiovascular morbidity in patients with psoriatic arthritis: A meta-analysis of observational studies. Arthritis Care Res 2017;69:67-74.

6. O'Leary DH, Polak JF, Kronmal RA, Manolio TA, Burke GL, Wolfson SK Jr. Carotid-artery intima and media thickness as a risk factor for myocardial infarction and stroke in older adults. Cardiovascular Health Study Collaborative Research Group. N Engl J Med 1999;340:14-22.

7. Nambi V, Chambless L, Folsom AR, He M, Hu Y, Mosley T, et al. Carotid intima-media thickness and presence or absence of plaque improves prediction of coronary heart disease risk: the ARIC (Atherosclerosis Risk In Communities) study. J Am Coll Cardiol 2010;55:1600-7.

8. Corrales A, Gonzalez-Juanatey C, Peiro ME, Blanco R, Llorca J, Gonzalez-Gay MA. Carotid ultrasound is useful for the cardiovascular risk stratification of patients with rheumatoid arthritis: results of a population-based study. Ann Rheum Dis 2014;73:722-7.
9. Evans MR, Escalante A, Battafarano DF, Freeman GL, O'Leary $\mathrm{DH}$, del Rincon I. Carotid atherosclerosis predicts incident acute coronary syndromes in rheumatoid arthritis. Arthritis Rheum 2011;63:1211-20

10. Gonzalez-Juanatey C, Llorca J, Martin J, Gonzalez-Gay MA. Carotid intima-media thickness predicts the development of cardiovascular events in patients with rheumatoid arthritis. Semin Arthritis Rheum 2009;38:366-71.

11. Tam LS, Shang Q, Li EK, Tomlinson B, Chu TT, Li M, et al. Subclinical carotid atherosclerosis in patients with psoriatic arthritis. Arthritis Rheum 2008;59:1322-31.

12. Di Minno MN, Ambrosino P, Lupoli R, Di Minno A, Tasso M, Peluso R, et al. Cardiovascular risk markers in patients with psoriatic arthritis: A meta-analysis of literature studies. Ann Med 2015;47:346-53.

13. Tam LS, Tomlinson B, Chu TT, Li M, Leung YY, Kwok LW, et al. Cardiovascular risk profile of patients with psoriatic arthritis compared to controls - the role of inflammation. Rheumatology 2008;47:718-23.

14. Gonzalez-Juanatey C, Llorca J, Amigo-Diaz E, Dierssen T, Martin J, Gonzalez-Gay MA. High prevalence of subclinical atherosclerosis in psoriatic arthritis patients without clinically evident cardiovascular disease or classic atherosclerosis risk factors. Arthritis Rheum 2007;57:1074-80.

15. Roubille C, Richer V, Starnino T, McCourt C, McFarlane A, Fleming $\mathrm{P}$, et al. The effects of tumour necrosis factor inhibitors, methotrexate, non-steroidal anti-inflammatory drugs and corticosteroids on cardiovascular events in rheumatoid arthritis, psoriasis and psoriatic arthritis: a systematic review and meta-analysis. Ann Rheum Dis 2015;74:480-9.

16. D'Agostino RB Sr, Vasan RS, Pencina MJ, Wolf PA, Cobain M, Massaro JM, et al. General cardiovascular risk profile for use in primary care: the Framingham Heart Study. Circulation 2008;117:743-53

17. Hippisley-Cox J, Coupland C, Vinogradova Y, Robson J, Minhas R, Sheikh A, et al. Predicting cardiovascular risk in England and Wales: prospective derivation and validation of QRISK2. BMJ 2008;336:1475-82.

18. Conroy RM, Pyorala K, Fitzgerald AP, Sans S, Menotti A, De Backer G, et al. Estimation of ten-year risk of fatal cardiovascular disease in Europe: the SCORE project. Eur Heart J 2003; 24:987-1003.

19. Goff DC Jr, Lloyd-Jones DM, Bennett G, Coady S, D'Agostino RB Sr, Gibbons R, et al. 2013 ACC/AHA guideline on the assessment of cardiovascular risk: a report of the American College of Cardiology/American Heart Association Task Force on Practice Guidelines. J Am Coll Cardiol 2014;63:2935-59.

20. Arts EE, Popa C, Den Broeder AA, Semb AG, Toms T, Kitas GD, et al. Performance of four current risk algorithms in predicting cardiovascular events in patients with early rheumatoid arthritis. Ann Rheum Dis 2015;74:668-74.

21. Corrales A, Parra JA, Gonzalez-Juanatey C, Rueda-Gotor J, Blanco $\mathrm{R}$, Llorca $\mathrm{J}$, et al. Cardiovascular risk stratification in rheumatic diseases: carotid ultrasound is more sensitive than Coronary Artery Calcification Score to detect subclinical atherosclerosis in patients with rheumatoid arthritis. Ann Rheum Dis 2013;72:1764-70.

22. Ozen G, Sunbul M, Atagunduz P, Direskeneli H, Tigen K, Inanc N. The 2013 ACC/AHA 10-year atherosclerotic cardiovascular disease risk index is better than SCORE and QRisk II in rheumatoid arthritis: is it enough? Rheumatology 2016;55:513-22.

23. Gomez-Vaquero C, Corrales A, Zacarias A, Rueda-Gotor J, Blanco R, Gonzalez-Juanatey C, et al. SCORE and REGICOR function charts underestimate the cardiovascular risk in Spanish patients with rheumatoid arthritis. Arthritis Res Ther 2013;15:R91.

24. Kawai VK, Chung CP, Solus JF, Oeser A, Raggi P, Stein CM. The

Personal non-commercial use only. The Journal of Rheumatology Copyright (c) 2018. All rights reserved. 
ability of the 2013 American College of Cardiology/American Heart Association cardiovascular risk score to identify rheumatoid arthritis patients with high coronary artery calcification scores. Arthritis Rheumatol 2015;67:381-5.

25. Peters MJ, Symmons DP, McCarey D, Dijkmans BA, Nicola P, Kvien TK, et al. EULAR evidence-based recommendations for cardiovascular risk management in patients with rheumatoid arthritis and other forms of inflammatory arthritis. Ann Rheum Dis 2010;69:325-31.

26. Arts EE, Popa CD, Den Broeder AA, Donders R, Sandoo A, Toms $\mathrm{T}$, et al. Prediction of cardiovascular risk in rheumatoid arthritis: performance of original and adapted SCORE algorithms. Ann Rheum Dis 2016;75:674-80.

27. Ernste FC, Sanchez-Menendez M, Wilton KM, Crowson CS, Matteson EL, Maradit Kremers H. Cardiovascular risk profile at the onset of psoriatic arthritis: a population-based cohort study. Arthritis Care Res 2015;67:1015-21.

28. Eder L, Chandran V, Gladman DD. The Framingham Risk Score underestimates the extent of subclinical atherosclerosis in patients with psoriatic disease. Ann Rheum Dis 2014;73:1990-6.

29. Gulati AM, Semb AG, Rollefstad S, Romundstad PR, Kavanaugh A, Gulati S, et al. On the HUNT for cardiovascular risk factors and disease in patients with psoriatic arthritis: population-based data from the Nord-Trondelag Health Study. Ann Rheum Dis 2016;75:819-24

30. Taylor W, Gladman D, Helliwell P, Marchesoni A, Mease P, Mielants $\mathrm{H}$, et al. Classification criteria for psoriatic arthritis: development of new criteria from a large international study. Arthritis Rheum 2006;54:2665-73.

31. Shen J, Shang Q, Li EK, Leung YY, Kun EW, Kwok LW, et al. Cumulative inflammatory burden is independently associated with increased arterial stiffness in patients with psoriatic arthritis: a prospective study. Arthritis Res Ther 2015;17:75.

32. Wong PC, Leung YY, Li EK, Tam LS. Measuring disease activity in psoriatic arthritis. Int J Rheumatol 2012;2012:839425.

33. Mease PJ. Measures of psoriatic arthritis: Tender and Swollen Joint Assessment, Psoriasis Area and Severity Index (PASI), Nail Psoriasis Severity Index (NAPSI), Modified Nail Psoriasis Severity Index (mNAPSI), Mander/Newcastle Enthesitis Index (MEI), Leeds Enthesitis Index (LEI), Spondyloarthritis Research Consortium of Canada (SPARCC), Maastricht Ankylosing Spondylitis Enthesis Score (MASES), Leeds Dactylitis Index (LDI), Patient Global for Psoriatic Arthritis, Dermatology Life Quality Index (DLQI), Psoriatic Arthritis Quality of Life (PsAQOL), Functional Assessment of Chronic Illness Therapy-Fatigue (FACIT-F), Psoriatic Arthritis Response Criteria (PsARC), Psoriatic Arthritis Joint Activity Index (PsAJAI), Disease Activity in Psoriatic Arthritis (DAPSA), and Composite Psoriatic Disease Activity Index (CPDAI). Arthritis Care Res 2011;63 Suppl 11:S64-85.

34. Schoels MM, Aletaha D, Alasti F, Smolen JS. Disease activity in psoriatic arthritis (PsA): defining remission and treatment success using the DAPSA score. Ann Rheum Dis 2016;75:811-8.

35. Tam LS, Shang Q, Li EK, Wong S, Li RJ, Lee KL, et al. Serum soluble receptor for advanced glycation end products levels and aortic augmentation index in early rheumatoid arthritis - a prospective study. Semin Arthritis Rheum 2013;42:333-45.
36. Shen J, Shang Q, Wong CK, Li EK, Wang S, Li RJ, et al. IL-33 and soluble ST2 levels as novel predictors for remission and progression of carotid plaque in early rheumatoid arthritis: a prospective study. Semin Arthritis Rheum 2015;45:18-27.

37. Yu CM, Zhang Q, Lam L, Lin H, Kong SL, Chan W, et al. Comparison of intensive and low-dose atorvastatin therapy in the reduction of carotid intimal-medial thickness in patients with coronary heart disease. Heart 2007;93:933-9.

38. Chiu M, Austin PC, Manuel DG, Tu JV. Comparison of cardiovascular risk profiles among ethnic groups using population health surveys between 1996 and 2007. CMAJ 2010;182:E301-10.

39. Sheth T, Nair C, Nargundkar M, Anand S, Yusuf S. Cardiovascular and cancer mortality among Canadians of European, south Asian and Chinese origin from 1979 to 1993: an analysis of 1.2 million deaths. CMAJ 1999;161:132-8.

40. Libby P. Inflammation in atherosclerosis. Nature 2002;420:868-74.

41. Shen J, Shang Q, Tam LS. Targeting inflammation in the prevention of cardiovascular disease in patients with inflammatory arthritis. Transl Res 2016;167:138-51.

42. Ross R. Atherosclerosis - an inflammatory disease. N Engl J Med 1999;340:115-26.

43. Ridker PM, Buring JE, Rifai N, Cook NR. Development and validation of improved algorithms for the assessment of global cardiovascular risk in women: the Reynolds Risk Score. JAMA 2007;297:611-9.

44. Ridker PM, Paynter NP, Rifai N, Gaziano JM, Cook NR. C-reactive protein and parental history improve global cardiovascular risk prediction: the Reynolds Risk Score for men. Circulation 2008;118:2243-51

45. Dessein PH, Corrales A, Lopez-Mejias R, Solomon A, Woodiwiss AJ, Llorca J, et al. The Framingham Score and the Systematic Coronary Risk Evaluation at Low Cutoff Values Are Useful Surrogate Markers of High-risk Subclinical Atherosclerosis in Patients with Rheumatoid Arthritis. J Rheumatol 2016;43:486-94.

46. Chambless LE, Heiss G, Folsom AR, Rosamond W, Szklo M, Sharrett AR, et al. Association of coronary heart disease incidence with carotid arterial wall thickness and major risk factors: the Atherosclerosis Risk in Communities (ARIC) Study, 1987-1993. Am J Epidemiol 1997;146:483-94.

47. Salonen JT, Salonen R. Ultrasonographically assessed carotid morphology and the risk of coronary heart disease. Arterioscler Thromb 1991;11:1245-9.

48. Simon A, Chironi G, Levenson J. Comparative performance of subclinical atherosclerosis tests in predicting coronary heart disease in asymptomatic individuals. Eur Heart J 2007;28:2967-71.

49. Den Ruijter HM, Peters SA, Anderson TJ, Britton AR, Dekker JM, Eijkemans MJ, et al. Common carotid intima-media thickness measurements in cardiovascular risk prediction: a meta-analysis. JAMA 2012;308:796-803.

50. Inaba Y, Chen JA, Bergmann SR. Carotid plaque, compared with carotid intima-media thickness, more accurately predicts coronary artery disease events: a meta-analysis. Atherosclerosis 2012;220:128-33. 